\title{
Novel cationic lipid nanoparticles as an ophthalmic delivery system for multicomponent drugs: development, characterization, in vitro permeation, in vivo pharmacokinetic, and molecular dynamics studies
}

This article was published in the following Dove Press journal:

International Journal of Nanomedicine

6 November 2017

Number of times this article has been viewed

\author{
Jialu Wang' \\ Fang Zhao' \\ Rui Liu' \\ Jingjing Chen' \\ Qinghua Zhang' \\ Ruijuan Lao' \\ Ze Wang' \\ Xin Jin ${ }^{2}$ \\ Changxiao $\mathrm{Liu}^{3}$ \\ 'School of Chinese Materia Medica, \\ Tianjin University of Traditional \\ Chinese Medicine, Nankai District, \\ ${ }^{2}$ Department of Pharmacology, \\ Logistics University of Chinese \\ People's Armed Police Force, Dongli \\ District, ${ }^{3}$ State Key Laboratory \\ of Drug Delivery Technology and \\ Pharmacokinetics, Tianjin Institute \\ of Pharmaceutical Research, Nankai \\ District, Tianjin, China
}

Correspondence: Rui Liu

School of Chinese Materia Medica,

Tianjin University of Traditional Chinese

Medicine, 312 Anshan West Road,

Nankai District, Tianjin 300193, China

Email Ir_8000@I63.com

Changxiao Liu

State Key Laboratory of Drug Delivery

Technology and Pharmacokinetics,

Tianjin Institute of Pharmaceutical

Research, 308 Anshan West Road,

Nankai District, Tianjin 300193, China

Email liuchangxiao@I63.com

\begin{abstract}
The purpose of this study was to prepare, optimize, and characterize a cationic lipid nanoparticle (CLN) system containing multicomponent drugs using a molecular dynamics model as a novel method of evaluating formulations. Puerarin (PUE) and scutellarin (SCU) were used as model drugs. CLNs were successfully prepared using melt-emulsion ultrasonication and low temperature-solidification technique. The properties of CLNs such as morphology, particle size, zeta potential, entrapment efficiency (EE), drug loading (DL), and drug release behavior were investigated. The CLNs were evaluated by corneal permeation, preocular retention time, and pharmacokinetics in the aqueous humor. Additionally, a molecular dynamics model was used to evaluate the formulation. Electron microscopy results showed that the nanoparticles were approximately spherical in shape. The EE (\%) and DL (\%) values of PUE and SCU in the optimal formulation were $56.60 \pm 3.73,72.31 \pm 1.96$ and $1.68 \pm 0.17,2.44 \pm 1.14$, respectively. The pharmacokinetic study in the aqueous humor showed that compared with the PUE and SCU solution, the area under the concentration-time curve (AUC) value of PUE was enhanced by 2.33-fold for PUE-SCU CLNs $(p<0.01)$, and the SCU AUC was enhanced by 2.32 -fold $(p<0.01)$. In the molecular dynamics model, PUE and SCU passed through the POPC bilayer, with an obvious difference in the free energy well depth. It was found that the maximum free energy required for PUE and SCU transmembrane movement was $\sim 15$ and $88 \mathrm{~kJ} \cdot \mathrm{mol}^{-1}$, respectively. These findings indicated that compared with SCU, PUE easily passed through the membrane. The diffusion coefficient for PUE and SCU were $4.1 \times 10^{-3} \pm 0.0027$ and $1.0 \times 10^{-3} \pm 0.0006 \mathrm{e}^{-5} \mathrm{~cm}^{2} \cdot \mathrm{s}^{-1}$, respectively. Data from the molecular dynamics model were consistent with the experimental data. All data indicated that CLNs have a great potential for ocular administration and can be used as an ocular delivery system for multicomponent drugs. Moreover, the molecular dynamics model can also be used as a novel method for evaluating formulations.
\end{abstract}

Keywords: cationic lipid nanoparticle, molecular dynamics model, microdialysis, pharmacokinetic, preocular retention time, ocular delivery

\section{Plain language summary}

The purpose of this study was to prepare, optimize, and characterize a cationic lipid nanoparticle (CLN) system containing multicomponent drugs using a molecular dynamics model as a novel method of evaluating formulations. The properties of these CLNs such as morphology, particle size, zeta potential, entrapment efficiency, drug loading, and drug release behavior were investigated. 
The CLNs were evaluated by corneal permeation, preocular retention time, and pharmacokinetics in the aqueous humor. They have great potential for ocular administration and can be used as an ocular delivery system for multicomponent drugs. Moreover, the molecular dynamics model can also be used as a novel method for evaluating formulations.

\section{Introduction}

Nanoparticulate carrier systems (eg, lipid nanoparticles, liposomes, and microemulsions) have recently been under consideration for topical ophthalmic drug delivery as they have the potential to modulate drug release by facilitating drug transport to different compartments of the eye. ${ }^{1,2}$ These nanoparticles are characterized by a solid lipid core which is stabilized by surfactants in aqueous dispersion, are able to load lipophilic and hydrophilic drug molecules, and combine the advantages of other colloidal carriers, such as polymeric nanoparticles, fat emulsions, liposomes, or niosomes, ${ }^{3,4}$ avoiding their disadvantages. Ocular diseases are usually treated by topical application of drug solutions, such as eye drops. These conventional dosage forms account for nearly $90 \%$ of the currently available marketed formulations due to their simplicity, safety, and acceptance by patients. ${ }^{5}$ However, the physiological constraints imposed by the protective mechanisms of the eye and the limited permeability of the cornea lead to low absorption of ocular drugs and result in a short therapeutic effect. ${ }^{6}$ In addition, drugs exit the tear volume as tear fluid, which is continuously drained from the eyes to the nose during blinking, resulting in reduced bioavailability. ${ }^{7}$ Furthermore, most of the administered dose passes through the nasolacrimal duct into the gastrointestinal tract, which may cause side effects. ${ }^{8}$

Lipid nanoparticles have been reported to possess a number of positive effects as controlled nanodelivery systems. ${ }^{9}$ They are submicron colloidal carriers composed of biodegradable and biocompatible lipids that are generally recognized as safe. ${ }^{10,11}$ Lipid nanoparticles have the ability to penetrate cell membranes, allowing increased cellular uptake of the loaded compounds. ${ }^{12,13}$ The system used in the present study overcomes the disadvantages of the traditional lipid nanoparticle system, and can simultaneously contain multicomponent drugs and improve the drug entrapment efficiency (EE). Furthermore, octadecyl quaternized carboxymethyl chitosan (QACMC), a cationic material with good biocompatibility, biodegradability and nontoxic, plays an important role in prolonging drug efficacy, decreasing drug side effects, increasing drug absorption, and improving bioavailability. ${ }^{14-17}$
Some recent studies have focused on the in vivo fate of nanoparticles using various biosensing tools, such as fluorescent imaging, computed tomography, and magnetic resonance imaging. ${ }^{18}$ In this study, we used a noninvasive fluorescence imaging system and microdialysis techniques to analyze the in vivo fate of puerarin (PUE)-scutellarin (SCU) cationic lipid nanoparticles (CLNs).

PUE and SCU are used clinically to treat glaucoma and retinopathy. ${ }^{19,20}$ PUE is one of the important active components of Puerarin lobata (wild). It has the advantage of low toxicity and is quickly metabolized. However, its method of extraction and low solubility in lipid solvent or water due to its structure result in a poor bio-utilization ratio which restricts the bioactivity and clinic application of PUE. ${ }^{19,21}$ SCU is the main active compound in breviscapine. Breviscapine is a flavonoid extracted from Erigeron (Erigeron breviscapus (vant.)Hand.-Mazz). However, the solubility of breviscapine is poor in various solvents; it also has low oral bioavailability and a short half-life. ${ }^{20,22}$ PUE can reduce intraocular pressure, while SCU can protect the optic nerve and help to improve the optic nerve microcirculation. The concurrent application of these two drugs could be a focus in the treatment of both symptoms and the cause of ocular diseases.

Thus, it is necessary to develop a new PUE-SCU CLN system that not only increases the aqueous solubility of PUE and SCU, but also overcomes the shortcomings of conventional dosage forms. PUE-SCU CLNs were therefore characterized with respect to particle size (PS), zeta potential (ZP), EE, drug loading (DL), and particle morphology. In vitro drug release studies were performed using the dialysis bag method, and corneal permeation was also evaluated using excised rabbit corneas. Preocular retention capacity studies were conducted using a noninvasive fluorescence imaging system. A pharmacokinetic study in the aqueous humor was performed using a microdialysis technique. In addition, a molecular dynamics model of the drug was established in order to further evaluate the dosage form. To our knowledge, this is the first method which enhances ocular bioavailability following topical application of the CLN system containing multicomponent drugs.

\section{Materials and methods Materials}

PUE and SCU were purchased from Zelang Medical Technology Co, Ltd (98\% purity; Nanjing, Jiangsu, China). GMO (1-oleoyl-rac-glycerol) was purchased from Adamas Reagent Co, Ltd (Shanghai, China). F127 was provided 
by Fengli Jingqiu Commerce and Trade Co, Ltd (Beijing, China). Gelucire ${ }^{\circledR} 44 / 14$ was a gift from Gattefosse SA (SaintPriest, France). QACMC was supplied by Nantong Lvshen Biological Engineering Co, Ltd (Jiangsu, China). Lecithin was purchased from Solarbio Science \& Technology Co, Ltd (99\% purity; Beijing, China). Cholesterol was provided by Lanji Biological Company Co, Ltd (98\% purity; Shanghai, China). Tween ${ }^{\circledR} 80$ was provided by the Tianjin Chemical Reagent Company (Tianjin, China). All other chemicals and reagents were of analytical grade.

\section{Preparation of PUE-SCU-loaded CLNs}

GMO and SCU were dissolved in ethanol, and subsequently heated and stirred to form the first oil phase. Distilled water was the first aqueous phase. Thereafter, the first oil phase was dropped into the first aqueous phase and allowed to emulsify for 2 hours at $60^{\circ} \mathrm{C}$ using a magnetic heating stirrer to form the first emulsion. In addition, lecithin and cholesterol were dissolved in methanol and ethanol, at a ratio of $3: 1(\mathrm{w} / \mathrm{w})$, followed by the addition of Tween ${ }^{\circledR} 80$ to form the second oil phase. PUE, F127, QACMC, and 0.08\% (w/v) Gelucire ${ }^{\circledR} 44 / 14$ were dissolved in phosphate-buffered saline (PBS) to form the second aqueous phase. The second oil phase was dropped into the second aqueous phase and allowed to emulsify for 2 hours at $60^{\circ} \mathrm{C}$ using a magnetic heating stirrer to form the second emulsion. Then, the first emulsion was again dropped into the second emulsion, stirred for 5 minutes, and then cooled to room temperature. A further size reduction was achieved following 10-minute (using a 5 seconds on and 5 seconds off cycle) treatment in an ultrasonic cell pulverizer (JYD-250L; Zhixin Instruments, Shanghai, China) at $400 \mathrm{~W}$ to obtain a nanoemulsion.

\section{Characteristics of PUE-SCU-loaded CLNs Drug analysis}

Drug EE and DL were determined by ultrafiltration using centrifuge tubes fitted with an ultrafilter (Amicon Ultra, molecular weight cutoff $30 \mathrm{kDa}$; EMD Millipore, Billerica, MA, USA). Each formulation $(1,000 \mu \mathrm{L})$ was placed in a volumetric flask and then sonicated for 30 minutes (total drug). Approximately $500 \mu \mathrm{L}$ of each formulation was then placed in the upper chamber and centrifuged at 8,000 rpm for 10 minutes (free drug). Quantitative determination of free drug and total drug was performed using a Waters e2695 high-performance liquid chromatography (HPLC) system (Waters Corporation, Milford, MA, USA). The detector was set at $335 \mathrm{~nm}$. PUE and SCU were separated using a DikmaC18 column $(5 \mu \mathrm{m}, 4.6 \times 250 \mathrm{~mm})$, the column temperature was set at $30^{\circ} \mathrm{C}$, and gradient elution was performed under the set conditions. The mobile phase consisted of methanol and $0.2 \%$ acetic acid. The flow rate of the mobile phase was $1.0 \mathrm{~mL} \cdot \mathrm{min}^{-1}$. EE and DL were calculated according to Equations 1 and 2:

$$
\begin{gathered}
\mathrm{EE} \%=\frac{W_{\text {total }}-W_{\text {free }}}{W_{\text {total }}} \times 100 \\
\mathrm{DL} \%=\frac{\left(W_{\text {total }}-W_{\text {free }}\right) V}{\left(W_{\text {total }}-W_{\text {free }}\right) V+W_{\text {lipid }}} \times 100
\end{gathered}
$$

where $W_{\text {total }}, W_{\text {free }}$, and $W_{\text {lipid }}$ represent the total amount of drug in the nanoparticles, the amount of drug in the filtrate, and the amount of lipid in the formulation, respectively.

\section{PS and ZP analysis}

The PS, polydispersity index (PI), and ZP were determined using a Zetasizer (Nano-ZS; Malvern Instruments Ltd, Malvern, UK) after appropriate dilution with purified water at $25^{\circ} \mathrm{C}$.

\section{Morphological study}

The formulation was examined using a transmission electron microscope (TEM; HT7700; Hitachi Ltd, Tokyo, Japan). After diluting the sample 100-fold with purified water, one drop of the sample suspension was deposited onto a 300mesh coated carbon-copper grid, and the sample was allowed to settle for 3-5 minutes. Excess fluid was removed using absorbent paper.

\section{Central composite factorial design (CCD)}

During the preliminary study, the influence of each parameter on the physicochemical properties of PUE-SCU CLNs was assessed. To determine the optimal conditions for the technical procedure, 30 experimental runs were performed according to central composite design principles using Design-Expert 8.06 (Stat-Ease, Inc, Minneapolis, MN, USA) as described in Table 1. A four-factor, five-level central composite design was generated for optimization of the

Table I Independent variables and levels of experiment design

\begin{tabular}{llllll}
\hline Independent variables & \multicolumn{2}{l}{ Levels } \\
\cline { 2 - 6 } & $\mathbf{- 2}$ & $-\mathbf{I}$ & $\mathbf{0}$ & $\mathbf{I}$ & $\mathbf{2}$ \\
\hline $\mathrm{X}_{1}$ : amount of PUE $(\mathrm{mg})$ & 2 & 3.5 & 4 & 6.5 & 8 \\
$\mathrm{X}_{2}$ : amount of SCU (mg) & 3 & 5.25 & 6 & 9.75 & 12 \\
$\mathrm{X}_{3}$ : lecithin/cholesterol $(\%)$ & $\mathrm{I}$ & 3 & 5 & 7 & 9 \\
$\mathrm{X}_{4}:$ Tween ${ }^{\oplus 0}(\mathrm{~mL})$ & 0.1 & 0.575 & 2 & 1.525 & 2 \\
\hline
\end{tabular}

Abbreviations: PUE, puerarin; SCU, scutellarin. 
PUE-SCU-loaded CLNs preparation. The study design involved investigating the effects of four independent variables: the amount of PUE $\left(\mathrm{X}_{1}\right), \mathrm{SCU}\left(\mathrm{X}_{2}\right)$, lecithin/cholesterol ratio $\left(\mathrm{X}_{3}\right)$, and the amount of Tween ${ }^{\circledR} 80\left(\mathrm{X}_{4}\right)$. The dependent variables were EE $\left(\mathrm{Y}_{1}\right)$ and DL $\left(\mathrm{Y}_{2}\right)$ of PUE-SCU CLNs. The sign and value of the quantitative effect represented the tendency and magnitude of the responses, respectively. EE and DL were inputs for the construction of the respective response surface model (RSM).

\section{In vitro drug release evaluation}

In vitro drug release was evaluated using the dynamic dialysis bag method. ${ }^{23}$ Briefly, $2 \mathrm{~mL}$ each of PUE-SCU solution and the PUE-SCU CLNs formulation were separately loaded into dialysis bags. The dialysis bags were then immersed in $60 \mathrm{~mL}$ of freshly prepared PBS ( $\mathrm{pH} 6.8)$ maintained at a thermostatically controlled temperature of $34^{\circ} \mathrm{C} \pm 0.5^{\circ} \mathrm{C}$. The magnetic stirring speed was $200 \mathrm{rpm}$. The samples withdrawn were replaced with similar dissolution media. The percent drug release after $0.5,1,2,4,6,8,10$, and 12 hours was determined by HPLC using Equation 3.

$$
\mathrm{f}_{2}=50 \log _{10}\left\{1+\frac{100}{\sqrt{1+\frac{1 \sum_{i=1} W_{\mathrm{t}}\left(\overline{R_{\mathrm{t}}}-\overline{T_{\mathrm{t}}}\right)^{2}}{\mathrm{n}}}}\right\}
$$

\section{In vitro corneal permeation evaluation}

Corneal permeation studies were performed using excised rabbit corneas. ${ }^{24}$ Rabbits were overdosed with an injection of air into the marginal ear vein. Corneas were immediately excised and preserved in glutathione bicarbonate Ringer's solution. Each sample $(500 \mu \mathrm{L})$ was added to the donor chamber, and $4.5 \mathrm{~mL}$ of fresh PBS ( $\mathrm{pH}$ 6.8) was added to the receptor chamber. Samples from the receptor chamber were withdrawn at predetermined time intervals and replaced with an equal volume of fresh PBS ( $\mathrm{pH}$ 6.8) for 6 hours (40, 80, 120, 160, 200, 240 minutes). All analyses were conducted as described in the drug concentration evaluation. The experiments were performed in triplicate. The apparent corneal permeability coefficient $\left(P_{\text {app }}\right)$ was calculated using the following equation: ${ }^{25}$

$$
P_{\text {app }}=\frac{\Delta Q}{\Delta t \cdot C_{0} \cdot A \cdot 60}
$$

where $\frac{\Delta Q}{\Delta t}$ is the slope of the linear portion of the cumulative permeated amount versus time plot, $C_{0}$ is the initial drug concentration in the donor cell, $A$ is the exposed corneal surface area $\left(0.5 \mathrm{~cm}^{2}\right)$, and 60 is the conversion of minutes to seconds.

\section{Preocular retention time evaluation}

The preocular retention of PUE-SCU CLNs was assessed using a noninvasive fluorescence imaging system (Night OWL II LB985; Berthold Technologies, Bad Wildbad, Germany). The CLN formulation was labeled as follows: PUE and SCU were replaced with rhodamine B $(\mathrm{RhB})$ in the oil phase and then processed using the same method as for the CLN preparation. The albino rabbits were examined under conscious condition. Immediately before imaging, the animals were anesthetized with chloral hydrate (injection $2.5 \mathrm{~mL} \cdot \mathrm{kg}^{-1}$ ) via the ear vein. Precisely $20 \mu \mathrm{L}$ of the formulation was injected directly into the conjunctival sac of the right eye, and the left eye was used as a control (RhB solution). The eyes were manually closed for 10 seconds to allow distribution over the cornea. Imaging was performed at $0,15,30,60,90,120$, and 150 minutes. The region of interest (ROI) for residual fluorescence was around the ocular and non-ocular areas. ${ }^{26,27}$ The remaining intensity $(R)$ was calculated according to Equation 5:

$$
R=\frac{A-B}{C} \times 100 \%
$$

where $A$ is the intensity of the ROI, $B$ is the background fluorescence intensity, and $C$ is the intensity of the ROI at 0 minutes. ${ }^{26}$

\section{In vivo study}

\section{Animals}

New Zealand white rabbits weighing 2.5-3.0 kg were purchased from Tianjin Yuda Experimental Animal Culture Co, Ltd, Tianjin, China. The animals were housed at $25^{\circ} \mathrm{C} \pm 1^{\circ} \mathrm{C}$ and $50 \% \pm 5 \%$ relative humidity with ad libitum access to food and water. All animal experiments were reviewed and approved by the Animal Ethical Committee at Tianjin University of Traditional Chinese Medicine, and followed the animal care guidelines of the Laboratory Animal Management Committee.

\section{Pharmacokinetic study of the aqueous humor}

A pharmacokinetic study using the aqueous humor was performed by microdialysis. ${ }^{28}$ Different formulations were used to study the concentration of the drug in aqueous humor (formulation 1 was lipid nanoparticles, formulation 2 was lipid nanoparticles with QACMC as a cationic material, 
and formulation 3 was lipid nanoparticles with QACMC and Gelucire ${ }^{\circledR} 44 / 14$ as a cationic material and penetration enhancer, respectively). Briefly, three rabbits were anesthetized via an injection of $2.5 \mathrm{~mL} \cdot \mathrm{kg}^{-1}$ of chloral hydrate into the marginal ear vein. A microdialysis probe (MD 2000; Bioanalytical Systems, Inc, West Lafayette, IN, USA) was implanted into the anterior chamber of the eye using a $10 \mathrm{G}$ needle. The needle was removed, leaving the probe with the microdialysis membrane in the middle of the anterior chamber. Thereafter, the probe was perfused with PBS (pH 6.8) at a flow rate of $3 \mu \mathrm{L} \cdot \mathrm{min}^{-1}$ using a microdialysis pump (CMA106; CMA Microdialysis Co, Ltd, Stockholm, Sweden). The corneal wound surface was treated with $0.3 \%$ (w/v) ofloxacin ophthalmic drops and was stable for 24 hours before the formulations were administered. ${ }^{29}$ The dialysate was collected 20 minutes after 60 minutes of perfusion. In vivo recovery $(R)$ was calculated using Equation 6 :

$$
R \%=\frac{C_{\mathrm{d}}-C_{\mathrm{p}}}{C_{\mathrm{m}}-C_{\mathrm{p}}} \times 100
$$

where $C_{\mathrm{d}}, C_{\mathrm{p}}$, and $C_{\mathrm{m}}$ are the concentrations of the drug in the dialysate, perfusate, and aqueous humor, respectively. $R$ is the value of the slope for the plot of $\left(C_{\mathrm{d}}-C_{\mathrm{p}}\right)$ versus $C_{\mathrm{p}}$.

Therefore, $0.2 \mathrm{~mL}$ of each formulation $\left(1.0 \mathrm{mg} \cdot \mathrm{mL}^{-1}\right)$ was injected into different groups of rabbit eyes. Dialysates were collected every 20 minutes for the first 2 hours and then every 30 minutes after instillation. Each experiment was repeated three times, and all samples were analyzed using HPLC.

\section{The molecular dynamics model}

\section{System building}

As for the united-atom model, the model of Tieleman et $\mathrm{al}^{30}$ including 128 POPC molecules and 2,460 water molecules was used as the initial structure (http://moose.bio.ucalgary. ca/index.php?page=Structures and Topologies). The system was elongated $0.7 \mathrm{~nm}$ at the two ends of the z-axis. Thus, the whole system had 128 POPC molecules and 4,398 water molecules. The obtained system was equilibrated for 500 ps. PUE and SCU were optimized using a Gaussian program with the B3LYP/6-311+G $(d, p)$ method. The charge of each atom model was derived using the restrained electrostatic potential method at the B3LYP/6-311+G (d, p) level. The force field parameters were generated by the PRODRG ${ }^{31}$ server.

\section{Computation of the potential of mean force (PMF)}

The PMF method ${ }^{32,33}$ was used to calculate the free energy profile of PUE and SCU passing across the POPC bilayer. The calculations were performed using GROMACS 4.5.4.
This lipid bimolecular system contains 128 POPC molecules and 6,888 water molecules. The force field parameters of the POPC were obtained from the literature. ${ }^{33}$ PUE and SCU were first placed in the center of the bilayer, and they were pulled into bulk water along the $z$-axis using the umbrella sampling method. They were constrained at different $z$ locations with a restraint of $z$ distance between the center of mass of PUE, SCU, and the POPC bilayer. The harmonic restraint and pulling rate were set at $1,000 \mathrm{~kJ} \cdot \mathrm{mol}^{-1} \mathrm{~nm}^{-2}$ and 0.01 $\mathrm{nm} \cdot \mathrm{ps}^{-1}$, respectively. The molecular dynamics simulation was applied for $10 \mathrm{~ns}$. The force field used was the GROMOS96 53a6 force field. In addition, the free energy change curve of the drug permeation process was obtained by the weighted histogram analysis method. ${ }^{34,35}$ Free energy was calculated using Equation 7:

$$
\Delta G(z)=-\int_{z=\infty}^{z}<F(z, t)>_{\text {time }} d z
$$

\section{Molecular dynamics simulation}

Eight drug molecules were placed in the water on both sides of the POPC bilayer membrane and the water molecules near the drug molecules were removed. The energy was first minimized; then, 100 ps of NVT (volume:constant number) and NPT(constant temperature and constant pressure) were equilibrated for a 100-ns molecular dynamics simulation. The diffusion constant was calculated by least squares fitting a straight line through the mean square displacement from - beginfit to - endfit.

\section{Statistical analysis}

The optimization design of PUE-SCU CLNs and the resulting data were analyzed by Design-Expert software (8.06 version).The results are presented as mean \pm standard deviation. Statistical analysis of the results was performed using two-way analysis of variance (ANOVA) and one-way ANOVA using SPSS software (17.0 version; SPSS Inc, Chicago, IL, USA). Statistical significance was established at $p<0.05$.

\section{Results and discussion Central CCD}

After pre-dose optimization and selection of excipients, the amount of PUE, SCU, Tween ${ }^{\circledR} 80$, and the ratio of lecithin and cholesterol had a significant effect on EE and DL. Through the range of the single factor test combined with the results of effect surface, EE, and DL as the evaluation index, the optimal PUE-SCU CLNs formulation was obtained. 
A quadratic polynomial equation was used to represent the response with regard to the independent variables, as shown in Equation 8:

$$
\begin{aligned}
Y= & A_{0}+A_{1} X_{1}+A_{2} X_{2}+A_{3} X_{3}+A_{4} X_{4}+A_{5} X_{1} X_{2} \\
& +A_{6} X_{1} X_{3}+A_{7} X_{1} X_{4}+A_{8} X_{2} X_{3}+A_{9} X_{2} X_{4} \\
& +A_{10} X_{3} X_{4}+A_{11} X_{12}+A_{12} X_{22}+A_{13} X_{32}+A_{14} X_{42}
\end{aligned}
$$

where $\mathrm{Y}$ is the dependent variable, $\mathrm{X}_{1}-\mathrm{X}_{4}$ are the independent variables, and $\mathrm{A}_{1}-\mathrm{A}_{14}$ are the regression coefficients of the respective variables.

It was demonstrated that the best-fit model for the responses was the quadratic model, which had the maximum $r$-value. ANOVA was performed to evaluate the significance of the quadratic model terms on the response and quantitative effects, as shown in Table 2 . The respective $p$-values shown in Table 2 indicated that the effect of the lecithin/ cholesterol ratio $\left(\mathrm{X}_{3}\right)$ and the amount of Tween ${ }^{\circledR} 80\left(\mathrm{X}_{4}\right)$ had significant effects $(p<0.05)$ on the response. However, the amount of PUE $\left(\mathrm{X}_{1}\right)$ and $\mathrm{SCU}\left(\mathrm{X}_{2}\right)$ were not significant for the EE and DL.

The response surface was optimized for multivariate analysis and plotted in a three-dimensional model graph. The three-dimensional response surface plots for EE and DL are shown in Figure 1. Results from preliminary studies showed that the optimal parameters for the PUE-SCU

\begin{tabular}{|c|c|c|c|c|}
\hline \multirow[t]{2}{*}{ Parameters } & \multicolumn{4}{|c|}{ Regression coefficients } \\
\hline & $\mathrm{EE}_{1}(\%)$ & $\mathrm{DL}_{1}(\%)$ & $\mathrm{EE}_{2}(\%)$ & $\mathrm{DL}_{2}(\%)$ \\
\hline$A_{0}$ & 40.02 & 1.04 & 53.91 & 1.72 \\
\hline$A_{1}$ & 1.35 & 0.17 & -0.23 & -0.24 \\
\hline $\mathrm{A}_{2}$ & 0.076 & $-6.667 E-0.03$ & -2.55 & 0.34 \\
\hline $\mathrm{A}_{3}$ & -1.95 & -0.062 & $\mathrm{I} .46$ & -0.028 \\
\hline $\mathrm{A}_{4}$ & 9.10 & 0.22 & 11.61 & 0.42 \\
\hline $\mathrm{A}_{5}$ & 2.07 & 0.041 & 2.60 & -0.016 \\
\hline$A_{6}$ & 0.92 & $8.750 \mathrm{E}-0.03$ & 1.34 & 0.12 \\
\hline $\mathrm{A}_{7}$ & 1.26 & 0.033 & 0.84 & -0.060 \\
\hline $\mathrm{A}_{8}$ & -2.53 & -0.092 & -3.46 & -0.22 \\
\hline $\mathrm{A}_{9}$ & 1.97 & 0.12 & 2.44 & 0.28 \\
\hline$A_{10}$ & -0.87 & -0.11 & -1.68 & -0.072 \\
\hline$A_{11}$ & 1.53 & 0.043 & 1.52 & 0.10 \\
\hline$A_{12}$ & 1.19 & 0.021 & 0.47 & $9.167 \mathrm{E}-0.03$ \\
\hline$A_{13}$ & -2.15 & -0.095 & 1.65 & -0.13 \\
\hline $\mathrm{A}_{14}$ & -2.34 & -0.061 & -2.30 & -0.053 \\
\hline $\mathrm{R}$ & 85.98 & 88.38 & 94.09 & 92.22 \\
\hline$p$-value & 0.0204 & 0.0072 & 0.0001 & 0.0006 \\
\hline
\end{tabular}

Table 2 Analysis of variance of the regression coefficients of the quadratic equations

Note: $A_{0}-A_{14}$ : coefficient of independent variables.

Abbreviations: $\mathrm{EE}_{1}, \mathrm{EE}_{2}$, encapsulation efficiencies of puerarin and scutellarin; $\mathrm{DL}_{1}$, $D L_{2}$, drug loading of puerarin and scutellarin.
CLNs formulation were $9.75 \mathrm{mg}$ SCU, $6.5 \mathrm{mg}$ PUE, $80 \mathrm{mg}$ GMO, $1.5 \mathrm{~mL}$ Tween ${ }^{\circledR} 80,0.08 \%$ (w/v) Gelucire ${ }^{\circledR} 44 / 14,30$ mg QACMC, and a ratio of lecithin to cholesterol of $3: 1$ $(w / w)$. The EE and DL values of the optimal formulation were $56.60 \pm 3.73,72.31 \pm 1.96$ and $1.68 \pm 0.17,2.44 \pm 1.14$, respectively. These findings indicate that most of the drugs were encapsulated in the liquid nanoparticles and that this formulation was considered to be the most suitable formulation for ophthalmic applications and used for further characterization.

Central CCD is an experimental design method and widely used to establish a second-order RSM in process optimization studies. ${ }^{36} \mathrm{CCD}$ is an effective alternative to full factorial design and enables the gathering of more data with a lower number of experiments. ${ }^{37}$

\section{PS, ZP, and morphology of PUE-SCU CLNs}

Appropriate PS and a narrow size range in the ophthalmic formulation can ensure low irritation, sufficient bioavailability, and good compatibility in ocular tissues. ${ }^{38}$ Therefore, the PS of the ophthalmic formulation has a significant effect on ophthalmic preparations. The mean diameter of the PUE-SCU CLNs was $181.0 \mathrm{~nm}$ with a PI of 0.224 (Figure 2A). The PS distribution is advantageous for uptake of PUE-SCU CLNs through the cornea. Ultrasonic shredding can change PS, but strong mechanical treatment may affect DL. The optimal formulation had a positive surface charge of $23.8 \mathrm{mV}$ (Figure 2B). Furthermore, the use of F127 as a stabilizer also has a desirable effect by increasing PUE-SCU CLNs stability. ${ }^{39}$ The results of TEM showed that the sample consisted of spherical particles with a smooth surface, as shown in Figure 2C.

\section{In vitro release of PUE-SCU CLNs}

In vitro drug release of PUE and SCU from PUE-SCU CLNs formulations was evaluated (Figure 3). In this study, PBS ( $\mathrm{pH}$ 6.8) was selected to enhance the solubility of PUE and SCU. It can be seen in Figure 3 that the diffusion of PUE from PUE-SCU CLNs was very rapid; $~ 52.35 \%$ of PUE was released in 1 hour, during which PUE exhibited an initial burst release. After the initial burst release, which lasted for $\sim 3$ hours, the release rate of PUE from CLNs slowed and was close to Higuchi release $(r=0.9840)$. After 12 hours, $\sim 95.48 \%$ of PUE had been released from the CLNs. However, with regard to SCU, 23.34\% of the drug was released in 1 hour. After 12 hours, $\sim 92.92 \%$ of SCU had been released from the CLNs. The release of SCU from the CLNs was also close to Higuchi release $(r=0.9720)$. Synchronized 
A

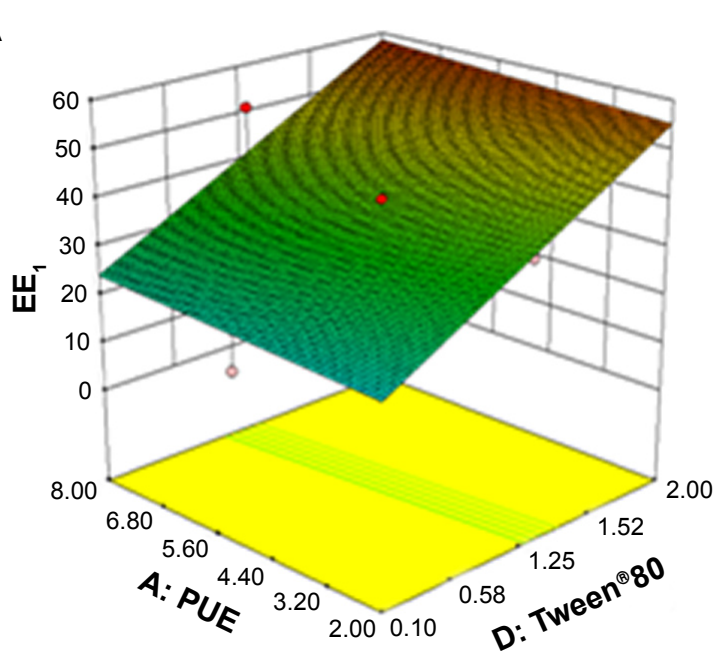

B

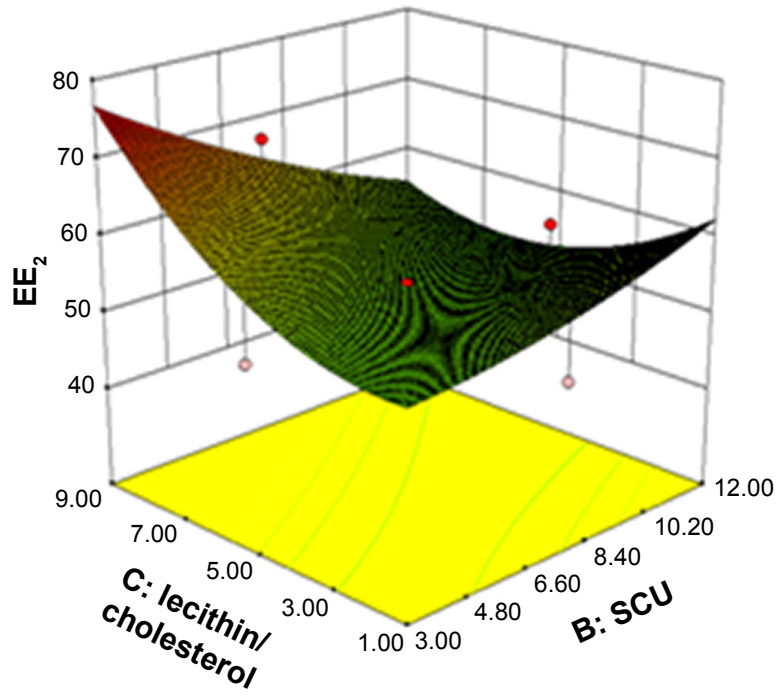

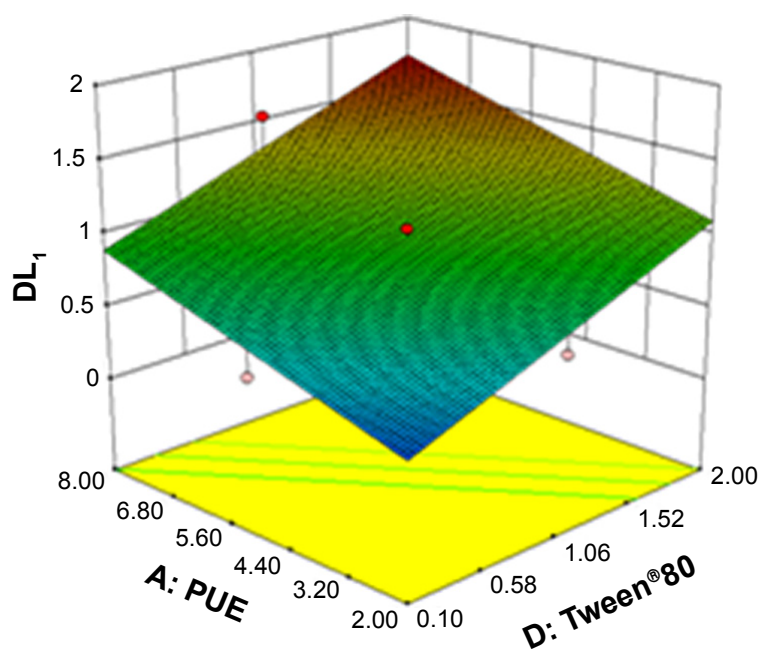

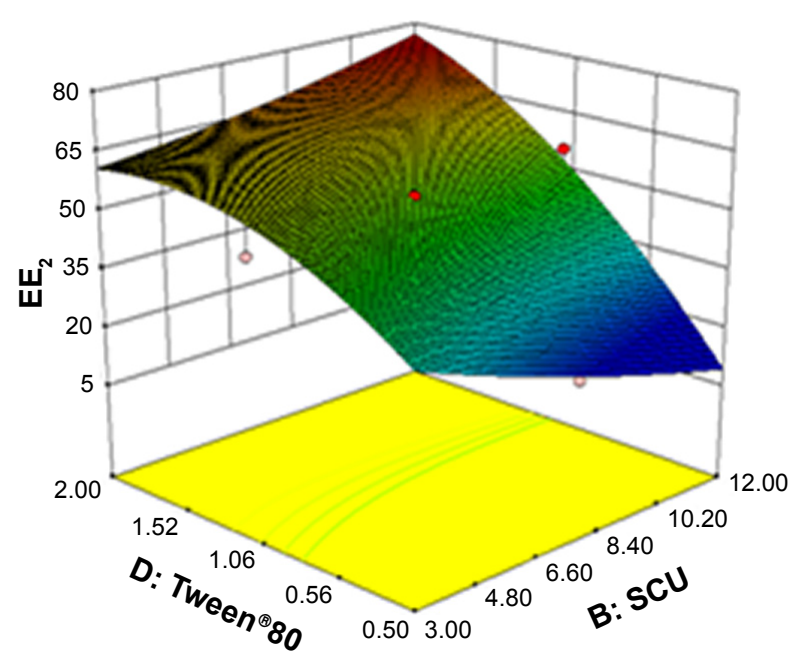

Figure I Three-dimensional response surface plots for EE and DL.

Notes: (A) Response surface plot showing the effect of the amount of PUE $\left(\mathrm{X}_{1}\right)$ and Tween ${ }^{\circledR} 80\left(\mathrm{X}_{4}\right)$ on the EE and DL of PUE (EE the effect of the amount of SCU $\left(X_{2}\right)$, Tween ${ }^{\circledR} 80\left(X_{4}\right)$, and SCU $\left(X_{2}\right)$, lecithin/cholesterol $\left(X_{3}\right)$ on the EE of SCU $\left(E_{2}\right)$.

Abbreviations: PUE, puerarin; SCU, scutellarin; EE, entrapment efficiency; DL, drug loading.

release was achieved with high similarity factor $\mathrm{f}_{2}$ values between each two of the five components. ${ }^{40}$ The US Food and Drug Administration demonstrated a simultaneous release similarity factor $>50 .{ }^{41}$ However, the similarity factor of PUE and SCU was 42.08. Therefore, the PUE-SCU CLNs demonstrated asynchronous release.

The reason was that PUE has better water solubility than that of SCU, while SCU was a liposoluble component. This asynchronous release showed that PUE released first, and played a role in reducing intraocular pressure. SCU released slowly, possessing optic nerve protection and microcirculation improvement effect. The concurrent application of these two drugs could be a focus in the treatment of both symptoms and the cause of ocular diseases. These results were in accordance with our experimental design. Additionally, the release rate of water-soluble drugs is higher than that of lipid-soluble drugs. ${ }^{42}$ This may be due to PUE with added nonionic surfactant, which can promote penetration, resulting in asynchronous release.

\section{In vitro corneal penetration evaluation}

In the corneal penetration study, the penetration of PUE and SCU through the cornea was examined when PUE and SCU solution or the same concentration of formulation was administered. CLNs can increase PUE and SCU penetration across the cornea. The $P_{\text {app }}$ values of the PUE solution and PUE-SCU CLNs were $7.11 \times 10^{-5}$ and $14.30 \times 10^{-5} \mathrm{~cm} \cdot \mathrm{s}^{-1}$, and the $P_{\text {app }}$ values of the SCU solution and PUE-SCU CLNs were $4.65 \times 10^{-6}$ and $5.72 \times 10^{-6} \mathrm{~cm} \cdot \mathrm{s}^{-1}$, respectively. Compared with the PUE and SCU solutions, PUE-SCU CLNs exhibited 
A
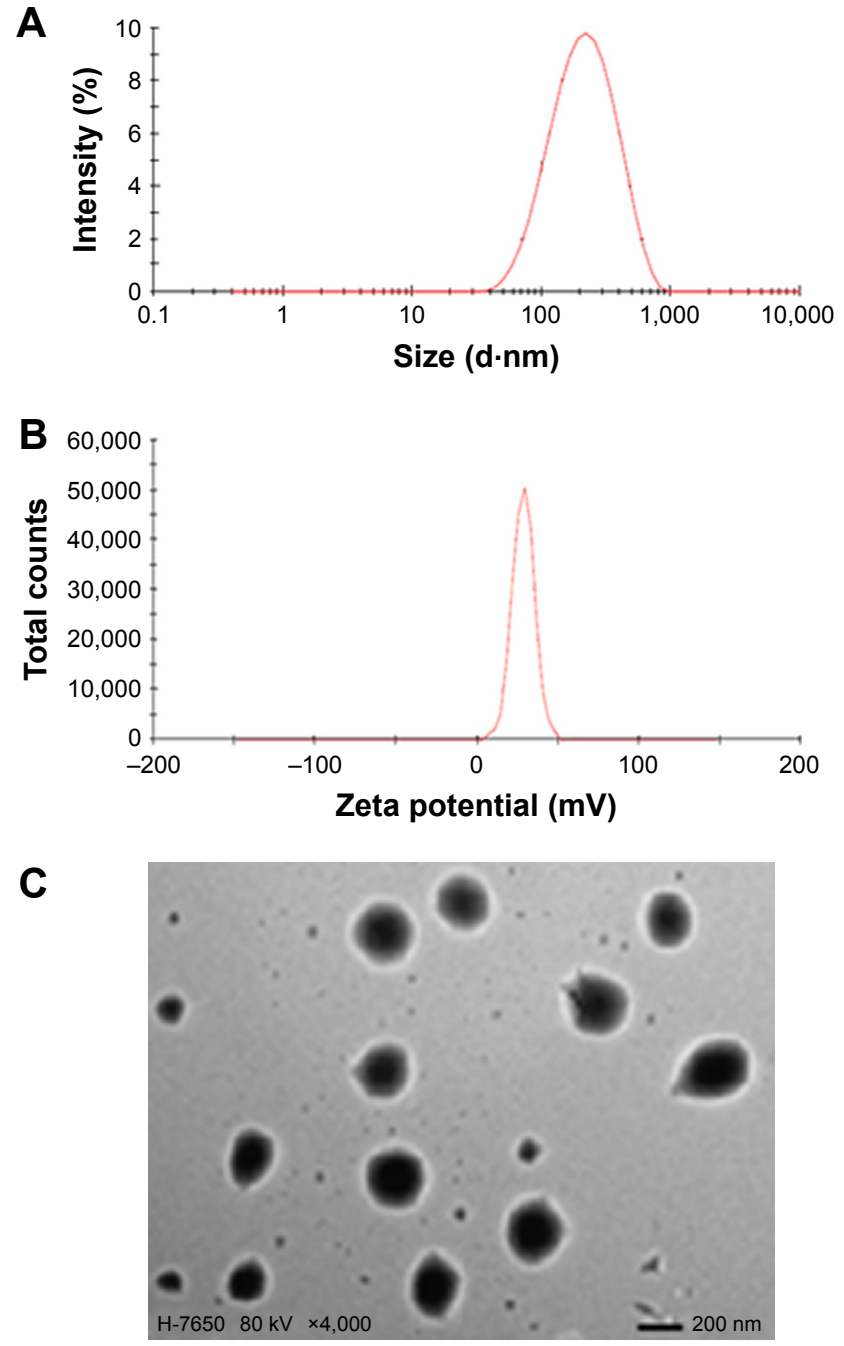

Figure 2 (A) Particle size distribution, (B) zeta potential distribution, and (C) transmission electron micrograph.

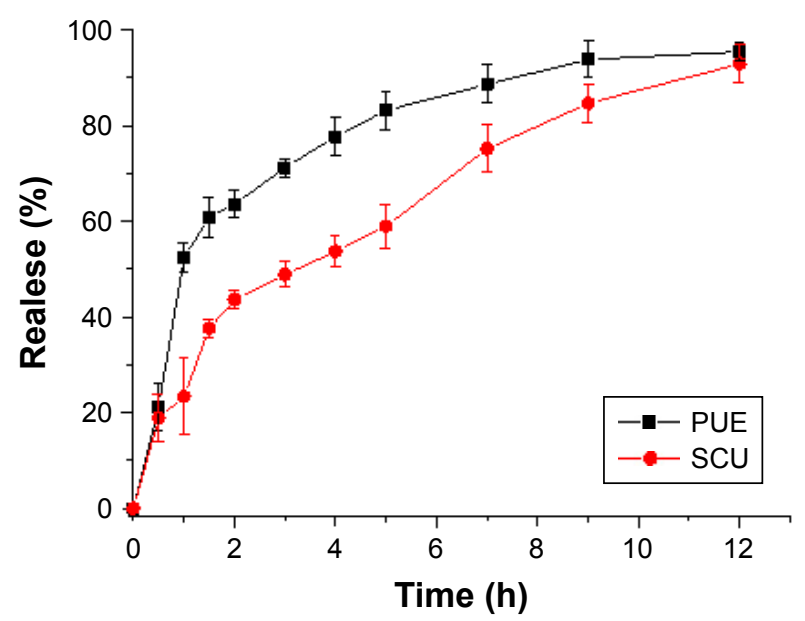

Figure 3 In vitro release profiles of PUE and SCU from PUE-SCU CLN formulations $(n=3)$.

Abbreviations: PUE, puerarin; SCU, scutellarin; CLN, cationic lipid nanoparticle. a 2.01-fold and 1.23-fold increase in $P_{\text {app}}$, suggesting that more PUE-SCU CLNs were taken up by the rabbit cornea compared with the PUE and SCU solutions. As the residence volume of the intraocular area is generally $7-10 \mathrm{~mL}$, most topically administered solutions are washed away within 15-30 seconds of application. ${ }^{43}$

One potential mechanism for enhancing corneal permeation was cationic material modification of lipid-based nanoparticles, which has been found to improve spreading properties, reduce contact angles, and increase residence time on the ocular surface, possibly interacting with the lipid layer of the tear film, so that the carrier can be retained in the conjunctival sac for a long period, where it acts as a drug depot. ${ }^{5,44}$ Another potential mechanism was that the liquid nanoparticles possess a bioadhesive property due to extremely small PS and increased surface area, which could promote permeation of the drug. Moreover, Gelucire ${ }^{\circledR} 44 / 14$ has been reported to be an effective corneal permeation enhancer. ${ }^{45}$ Thus, the combined effects of CLNs and Gelucire ${ }^{\circledR} 44 / 14$ may have significantly improved corneal penetration.

\section{Preocular retention of CLNs}

As shown in Figure 4A, rapid clearance was observed in the $\mathrm{RhB}$ solution group after administration. At 1.5 hours, only $50.22 \%$ fluorescence intensity remained in the ROI, which was significantly lower than that of RhB-CLNs which was $72.02 \%$. After 1.5 hours, statistical analysis showed that $34.63 \%$ and $60.52 \%$ intensity remained in the RhB solution and RhB-CLNs group, respectively. Up to 2.5 hours, relatively strong fluorescence intensity was still observed in the ROI in the RhB-CLNs group, as shown in Figure 4B. This indicated that the CLN formulation exhibited a relatively strong intensity and slow clearance from the ROI. The CLN preparation had greater viscosity, which increased the retention time on the cornea. In addition, the preparation was modified with the cationic material QACMC to form a positively charged carrier to further increase the retention time. The viscosity of the aqueous phase was increased thus hindering rapid dispersion from the corneal surface, and the electrostatic interaction between the cationic nanocarrier and the anionic surface of the cornea can reduce the contact angle and improve the drug residence. ${ }^{46,47}$

\section{Pharmacokinetic study in the aqueous humor}

In vivo probe recovery was determined before the pharmacokinetic study to ensure that the implanted probes functioned well. 

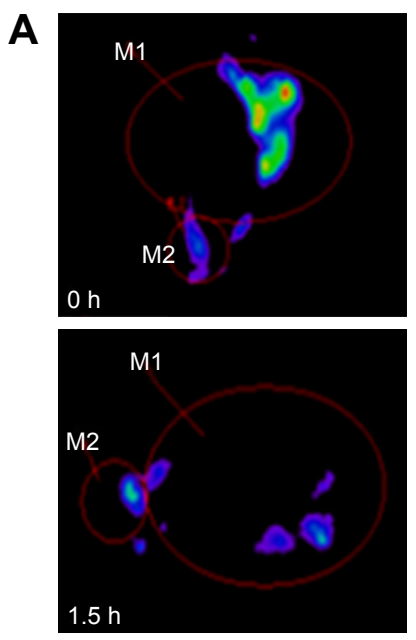
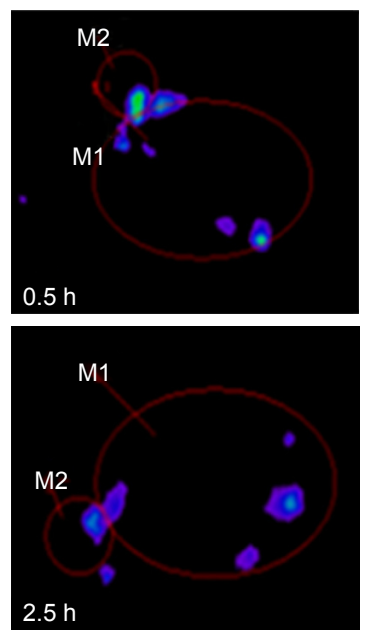

B
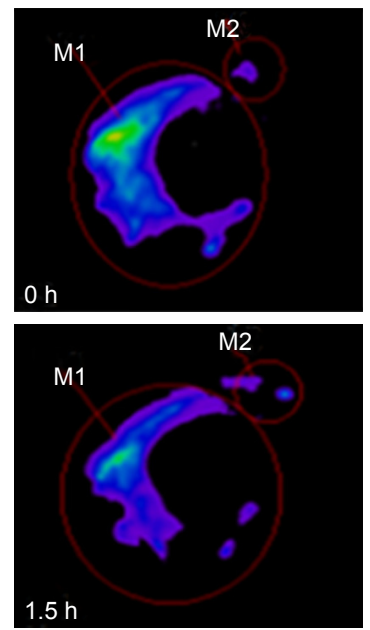
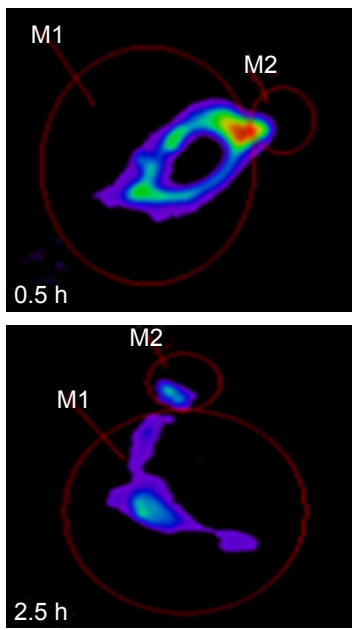

Figure 4 Fluorescence images of rabbit eyes after administration (MI: corneal surface; M2: inner canthus and nasolacrimal duct region). Notes: (A) RhB solution; (B) RhB-CLNs.

Abbreviations: CLNs, cationic lipid nanoparticles; RhB, rhodamine $B$.

The probe recovery of PUE and SCU in PUE-SCU CLNs was $56.02 \% \pm 3.93 \%(n=3)$ and $57.22 \% \pm 4.7 \%(n=3)$, respectively. Aqueous humor pharmacokinetics parameters are summarized in Tables 3 and 4. As shown in Figure 5A, compared with the PUE solution, the area under the concentration-time curve (AUC) value was enhanced by 2.33-fold for PUE-SCU CLNs $(p<0.01)$, and concentration at the peak $\left(\mathrm{C}_{\max }\right)$ value of PUE-SCU CLNs was enhanced by 1.69 -fold $(p<0.05)$. The half-life time $\left(t_{1 / 2}\right)$ of PUE-SCU CLNs was longer than that of the PUE solution. As shown in Figure 5B, compared with the SCU solution, the AUC value was enhanced by 2.32-fold for PUE-SCU CLNs $(p<0.01)$, and $\mathrm{C}_{\max }$ value of PUE-SCU CLNs was enhanced by 1.12 -fold $(p<0.05)$. The half-life time $\left(t_{1 / 2}\right)$ of PUE-SCU CLNs was longer than that of the PUE solution. There were significant differences in the $\mathrm{C}_{\max }$ and AUC values of PUE-SCU CLNs compared with the corresponding values for the PUE and SCU solutions $(p<0.01)$.

The enhanced ocular bioavailability provided by the CLN delivery system may be attributed to three factors, the ocular contact time of the delivery system, drug permeability through the cornea, and higher DL capacity. First, the preocular retention study indicated that the CLN formulation exhibited prolonged residence in the ROI, which may improve the ocular contact time as discussed above. Second, the in vitro corneal penetration evaluation showed that the $P_{\text {app }}$ value of the PUE-SCU CLNs exhibited a 2.01-fold and 1.23-fold increase relative to the PUE and SCU solutions. Furthermore, Gelucire ${ }^{\circledR} 44 / 14$ increases the solubility of dissolved drugs and enhances the absorption of poorly soluble drugs, contributing to improved drug bioavailability. ${ }^{45}$ All of these factors ensure intimate contact with the epithelial mucosal surface of the eye, preventing tear washout and consequently providing sustained drug release and a prolonged drug retention time. Such a system may be more advantageous than conventional dosage forms of PUE and SCU due to increased solubility and bioavailability. A sustained effect could improve patient compliance and reduce the dose and associated side effects.

Table 3 Pharmacokinetic parameters of PUE in aqueous humor after topical administration in the conscious rabbits $(\bar{X} \pm$ SD, $\mathrm{n}=3)$

\begin{tabular}{lllllll}
\hline Preparation & $\mathbf{T}_{\max }(\mathbf{h})$ & $\mathbf{C}_{\max }\left(\mu \mathbf{g} \cdot \mathbf{m L}^{-1}\right)$ & AUC $\left(\mu \mathbf{g} \cdot \mathbf{m L}^{-1} \cdot \mathbf{h}\right)$ & $\mathbf{T}_{1 / 2} \mathbf{( h )}$ & MRT (h) & VRT (h) \\
\hline Solution & 0.33 & $0.49 \pm 0.12$ & $1.01 \pm 0.23$ & $0.53 \pm 0.16$ & $1.13 \pm 0.35$ & $0.81 \pm 1.43$ \\
Formulation I & 0.67 & $0.59 \pm 0.30^{*}$ & $2.95 \pm 0.25^{* *}$ & $0.12 \pm 0.21$ & $1.06 \pm 0.31$ & $1.68 \pm 1.28$ \\
Formulation 2 & 0.67 & $0.64 \pm 0.09^{*}$ & $2.22 \pm 0.36 * *$ & $0.13 \pm 0.22$ & $1.48 \pm 0.33$ & $1.68 \pm 0.65$ \\
Formulation 3 & 0.67 & $0.83 \pm 0.28^{*}$ & $2.35 \pm 0.35^{* *}$ & $1.92 \pm 0.06 * *$ & $1.23 \pm 0.24$ & $1.56 \pm 1.21$ \\
\hline
\end{tabular}

Notes: Solution was PUE and SCU mixed solution; formulation I was lipid nanoparticles; formulation 2 was lipid nanoparticles with QACMC as a cationic material; and formulation 3 was lipid nanoparticles with QACMC and Gelucire ${ }^{\circledR} 44 / 14$ as a cationic material and penetration enhancer, respectively. ${ }^{*} p<0.05$ versus PUE solution; ${ }^{* *} p<0.0$ I versus PUE solution.

Abbreviations: $T_{\max }$, time to peak concentration; $\mathrm{C}_{\max }$, maximum concentration of PUE; AUC, area under the curve; $T_{1 / 2}$, half-life time; $M R T$, average residence time; VRT, retention time variance; PUE, puerarin; SCU, scutellarin; SD, standard deviation; QACMC, octadecyl quaternized carboxymethyl chitosan. 
Table 4 Pharmacokinetic parameters of SCU in aqueous humor after topical administration in the conscious rabbits $(\bar{X} \pm \mathrm{SD}, \mathrm{n}=3)$

\begin{tabular}{lllllll}
\hline Preparation & $\mathbf{T}_{\max }(\mathbf{h})$ & $\mathbf{C}_{\max }\left(\mu \mathbf{g} \cdot \mathbf{m L}^{-1}\right)$ & AUC $\left(\mu \mathbf{g} \cdot \mathbf{m L}^{-1} \cdot \mathbf{h}\right)$ & $\mathbf{T}_{1 / 2}(\mathbf{h})$ & MRT (h) & VRT (h) \\
\hline Solution & 0.33 & $0.50 \pm 0.37$ & $0.72 \pm 0.21$ & $0.43 \pm 0.22$ & $0.65 \pm 0.28$ & $0.66 \pm 1.15$ \\
Formulation I & 0.67 & $0.57 \pm 0.05^{*}$ & $1.42 \pm 0.18^{* *}$ & $1.63 \pm 0.23 * *$ & $0.91 \pm 0.35$ & $1.43 \pm 0.98$ \\
Formulation 2 & 0.33 & $0.52 \pm 0.14^{*}$ & $1.09 \pm 0.23^{*}$ & $0.79 \pm 0.33^{*}$ & $1.50 \pm 0.18$ & $1.45 \pm 1.02$ \\
Formulation 3 & 0.67 & $0.56 \pm 0.28^{*}$ & $1.67 \pm 0.15^{* *}$ & $1.82 \pm 0.33^{* *}$ & $1.08 \pm 0.24$ & $1.39 \pm 0.53$ \\
\hline
\end{tabular}

Notes: Solution was PUE and SCU mixed solution; formulation I was lipid nanoparticles; formulation 2 was lipid nanoparticles with QACMC as a cationic material; and formulation 3 was lipid nanoparticles with QACMC and Gelucire ${ }^{\circledR} 44 / 14$ as a cationic material and penetration enhancer, respectively. ${ }^{*} p<0.05$ versus SCU solution; ${ }^{* *} p<0.0$ I versus SCU solution.

Abbreviations: $T_{\max }$, time to peak concentration; $C_{\max }$, maximum concentration of SCU; AUC, area under the curve; $T_{1 / 2}$, half-life time; MRT, average residence time; VRT, retention time variance; PUE, puerarin; SCU, scutellarin; SD, standard deviation; QACMC, octadecyl quaternized carboxymethyl chitosan.

\section{The molecular dynamics model}

\section{Computation of the PMF}

Figure 6A and B shows the free energy profiles of PUE and SCU passing across the POPC bilayer, and it can be seen that the shape of these two free energy profiles are, to some extent, alike. The obvious difference was the free energy well depth. It was calculated from the figure that the maximum free energy required for PUE transmembrane movement was $\sim 15 \mathrm{~kJ} \cdot \mathrm{mol}^{-1}$, and for SCU was $\sim 88 \mathrm{~kJ} \cdot \mathrm{mol}^{-1}$. These results indicated that compared with SCU, PUE passed easily through the membrane.

In addition, molecular modeling of the POPC bilayer system was performed to ensure that finite size effects were not being observed. Due to time and resource constraints, a 10-ns simulation was performed. Figure 6C-F shows the final structure of PUE and SCU at the highest and lowest energies, respectively. The results indicated that PUE was more likely to remain in the hydrophobic region. Due to PUE being an isoflavone, the structure of 4-glucosine makes it aqueous soluble. ${ }^{48}$

\section{Molecular dynamics simulation}

Figure 7 shows the mean square deviation of PUE and SCU at $100 \mathrm{~ns}$. The diffusion coefficient of PUE and SCU was $4.1 \times 10^{-3} \pm 0.0027$ and $1.0 \times 10^{-3} \pm 0.0006 \mathrm{e}^{-5} \mathrm{~cm}^{2} \cdot \mathrm{s}^{-1}$, respectively. These results showed that the diffusion coefficient of PUE was higher than that of SCU, indicating that PUE was more likely to improve bioavailability. It can be seen from the data that the molecular dynamics model was consistent with the experimental results.

The use of multiscale mathematical and computational models to study complex biological processes is becoming increasingly productive. Advances in modeling are enabling virtual experiments to explore and answer questions that are problematic to address in the wet-lab. ${ }^{49}$ As computer power and algorithm design continue to improve, molecular dynamics
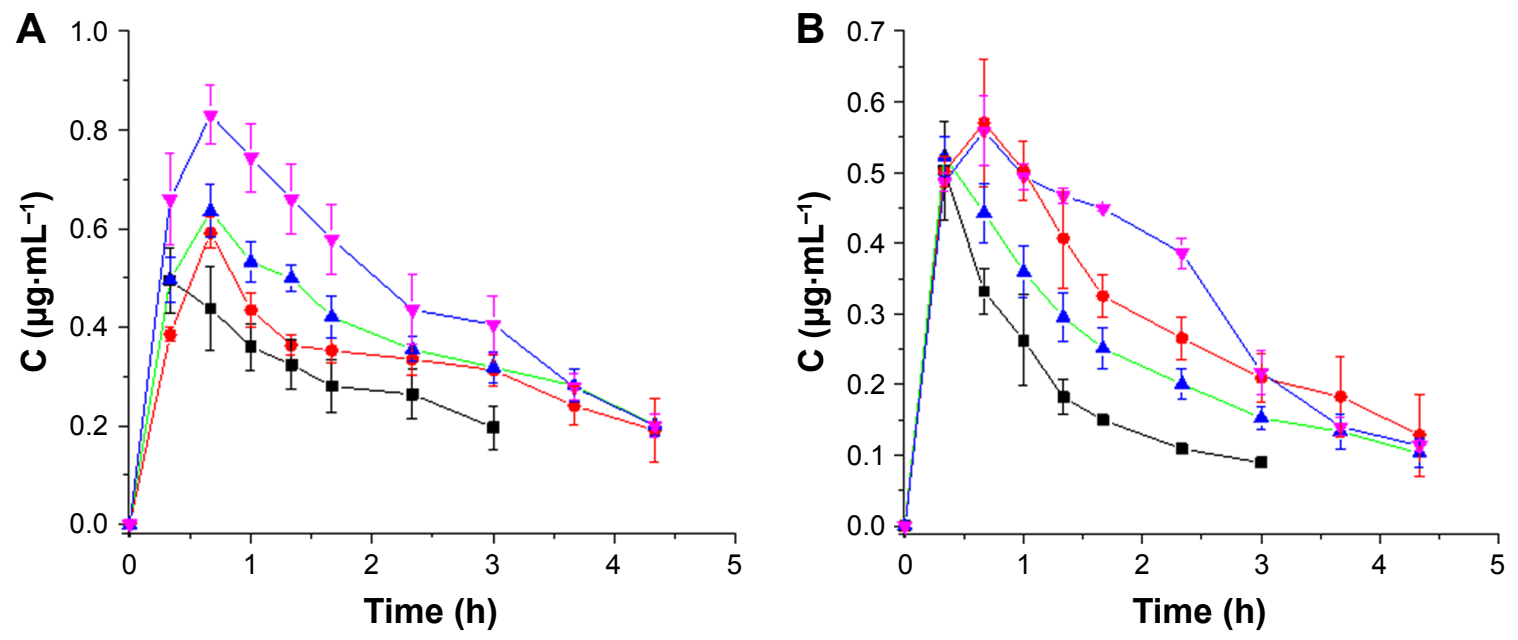

$-\mathbf{a}-$ Solution - Formulation $1-\boldsymbol{\Delta}$ - Formulation $2-\nabla-$ Formulation 3

Figure 5 PUE and SCU concentration-time profiles following a $200-\mu \mathrm{L}$ topical administration at a dose of $2.0 \mathrm{mg} \cdot \mathrm{mL}^{-1}$ in the aqueous humor ( $\mathrm{n}=3$ ).

Notes: (A) PUE; (B) SCU.

Abbreviations: PUE, puerarin; SCU, scutellarin. 
A

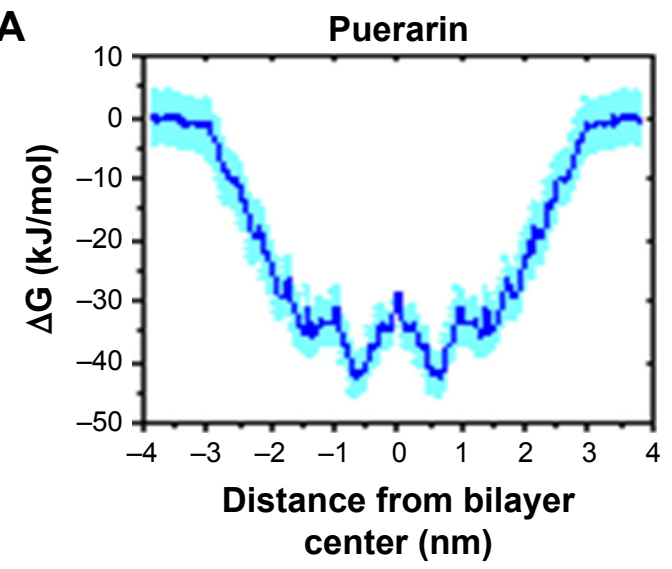

C

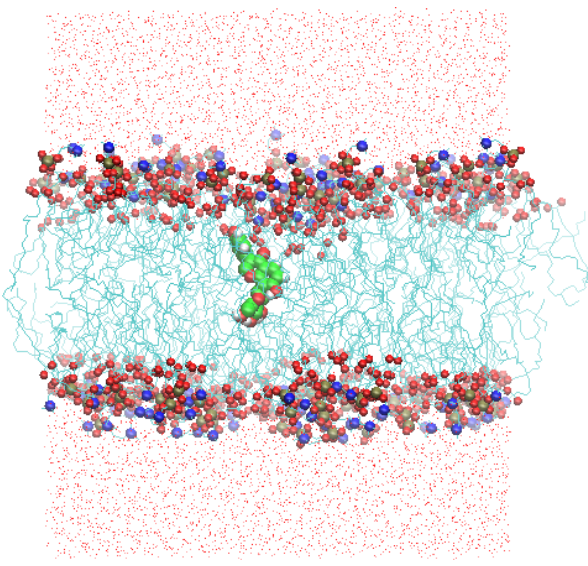

E

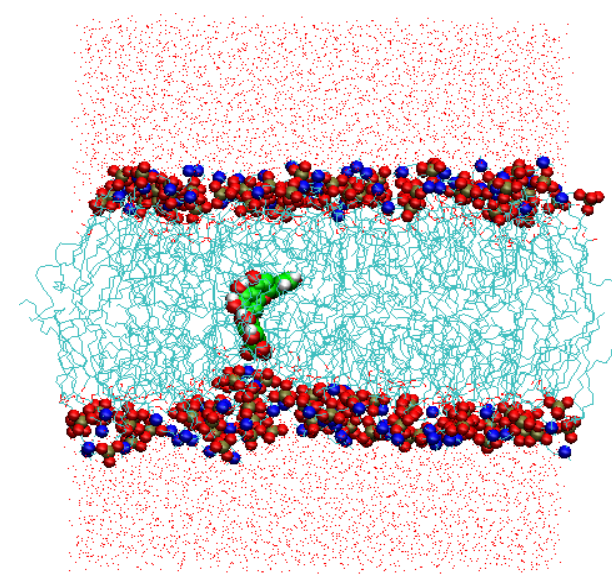

B

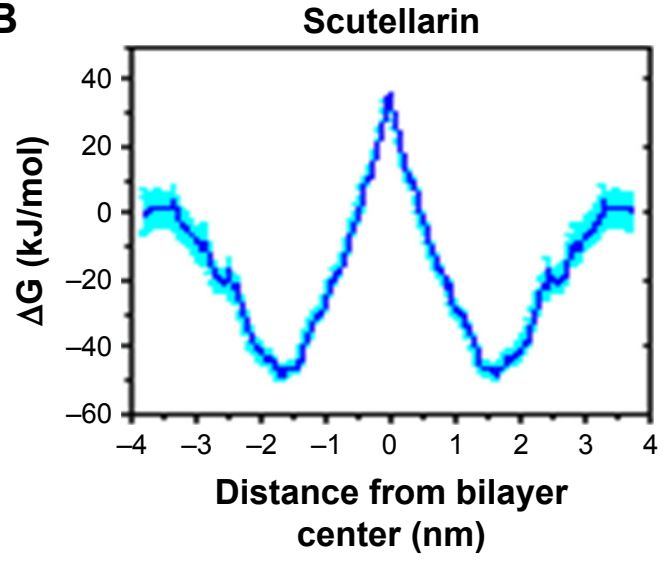

D

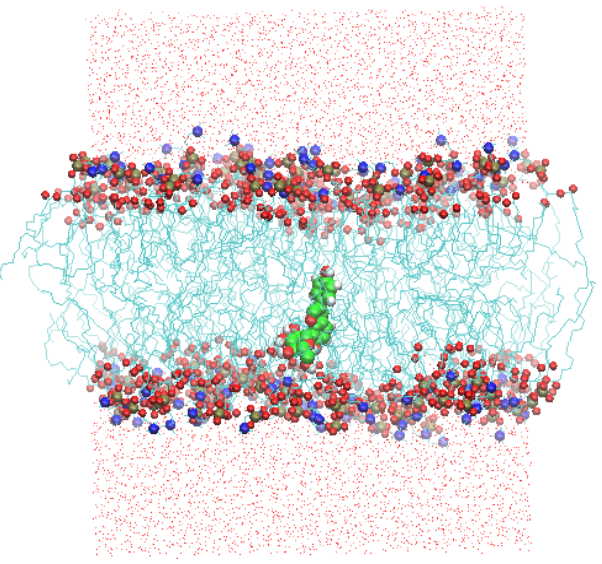

$\mathbf{F}$

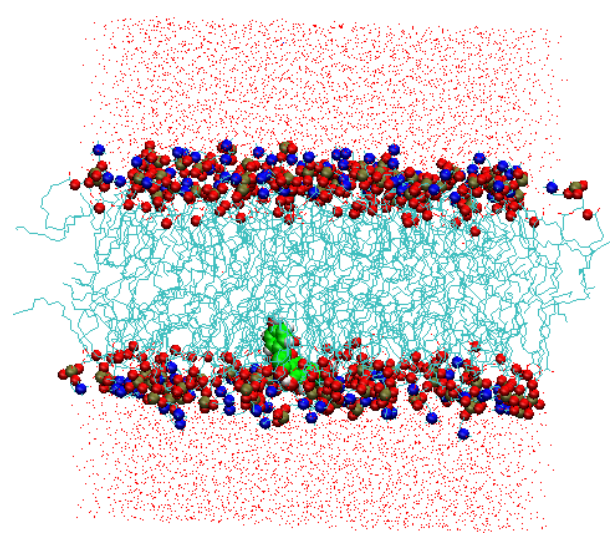

Figure 6 (A, B) Free energy profiles of PUE and SCU passing across POPC bilayer. (C, D) The final structure of PUE at the lowest and highest energies. (E, F) The final structure of SCU at the highest and lowest energies.

Abbreviations: PUE, puerarin; SCU, scutellarin.

simulation plays an increasingly important role. The molecular dynamics model is used in systems biology to understand, predict, and translate a wealth of experimentally generated data into a realization of systems behavior. This method has many advantages such as convenient modification of parameters, a short research period, and accurate and reliable results that are easy to verify. Modeling efforts are driving biological research from a descriptive field to a predictive field, especially in the context of pharmaceutical research. The ability to unify genomic, proteomic, and metabolomics data using modeling constructs is an emerging technique that facilitates new drug development and discovery along with new ways to repurpose old drugs. ${ }^{50,51}$ Therefore, molecular dynamics can be used as a new technique to evaluate formulations. 


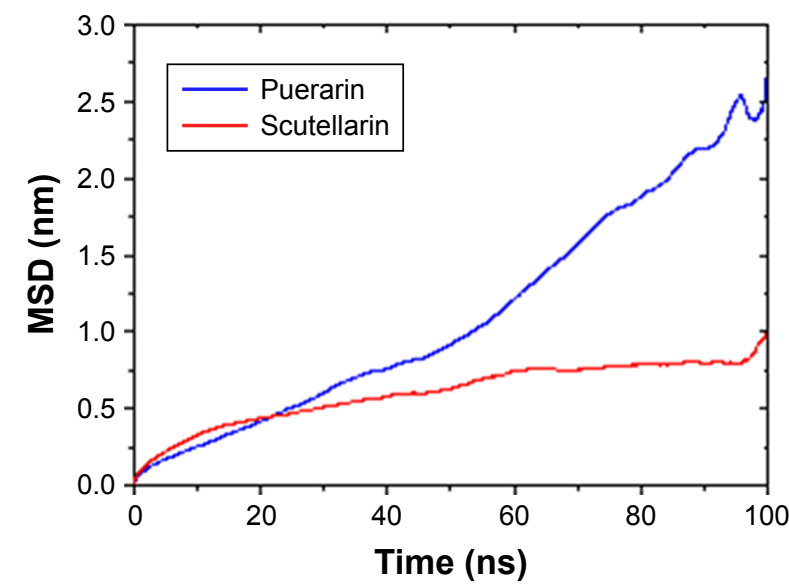

Figure 7 The MSD of PUE and SCU at 100 ns.

Abbreviations: PUE, puerarin; SCU, scutellarin; MSD, mean square deviation.

\section{Conclusion}

CLN systems containing multiple drug components can be used in ophthalmic drug delivery systems and can serve as a promising means of increasing the ocular bioavailability of PUE and SCU. Molecular dynamics is also a promising drug analysis method that can be used to evaluate the performance of formulations.

\section{Acknowledgments}

This work was funded by National Natural Science Foundation of China (No 81303142), National Science Foundation for Post-doctoral Researchers (No 2015M570231), Open Fund of Scientific Research Platform of Affiliated Hospital of Logistics University of Chinese People's Armed Police Force (WYKFM201609), Science and Technology Program of Tianjin (15PTCYSY00030), Program for Changjiang Scholars and Innovative Research Team in University (No IRT_14R41), and National Key Research and Development Program (No 2016YFE0121400).

\section{Disclosure}

The authors report no conflicts of interest in this work.

\section{References}

1. Badawi A, El-Laithy H, El Qidra R, El Mofty H, El Dally M. Chitosan based nanocarriers for indomethacin ocular delivery. Arch Pharm Res. 2008;31(8):1040-1049.

2. Balguri SP, Adelli GR, Majumdar S. Topical ophthalmic lipid nanoparticle formulations (SLN, NLC) of indomethacin for delivery to the posterior segment ocular tissues. Eur J Pharm Biopharm. 2016;109:224-235.

3. Li N, Zhuang C, Wang M, Sun X, Nie S, Pan W. Liposome coated with low molecular weight chitosan and its potential use in ocular drug delivery. Int J Pharm. 2009;379:131-138.

4. Abdelkader H, Ismail S, Amal K, Alan RG. Design and evaluation of controlled-release niosomes and discomes for naltrexone hydrochloride ocular delivery. J Pharm Sci. 2011;100:1833-1846.
5. Gan L, Wang J, Jiang M, et al. Recent advances in topical ophthalmic drug delivery with lipid-based nanocarriers. Drug Discov Today. 2013; 18(5-6):290-297.

6. Liu ZD, Zhang XH, Wu HY, et al. Preparation and evaluation of solid lipid nanoparticles of baicalin for ocular drug delivery system in vitro and in vivo. Drug Dev Ind Pharm. 2011;37(4):475-481.

7. Gause S, Hsu KH, Shafor C, Dixon P, Powell KC, Chauhan A. Mechanistic modeling of ophthalmic drug delivery to the anterior chamber by eye drops and contact lenses. Adv Colloid Interface Sci. 2016;233: 139-154.

8. Achouri D, Alhanout K, Piccerelle P, Andrieu V. Recent advances in ocular drug delivery. Drug Dev Ind Pharm. 2013;39(11):1599-1617.

9. Müller RH, Mäder K, Gohla S. Solid lipid nanoparticles (SCLN) for controlled drug delivery - a review of the state of the art. Eur J Pharm Biopharm. 2000;50(1):161-177.

10. Hentschel A, Gramdorf S, Müller RH, Kurz T. Beta-carotene-loaded nanostructured lipid carriers. J Food Sci. 2008;73(2):N1-N6.

11. Muchow M, Maincent P, Muller RH. Lipid nanoparticles with a solid matrix (SLN, NLC, LDC) for oral drug delivery. Drug Dev Ind Pharm. 2008;34(12):1394-1405.

12. Acosta E. Bioavailability of nanoparticles in nutrient and nutraceutical delivery. Curr Opin Colloid Interface Sci. 2009;14(1):3-15.

13. Balguri SP, Adelli G, Bhagav P, Repka MA, Majumdar S. Development of nano structured lipid carriers of ciprofloxacin for ocular delivery: characterization, in vivo distribution and effect of PEGylation. Invest Ophthalmol Vis Sci. 2015;56(7):2269.

14. Campo L, Yaghmur A, Sagalowicz L, Leser ME, Watzke H, Glatter O. Reversible phase transitions in emulsified nanostructured lipid systems. Langmuir. 2004;20:5254-5261.

15. Yaghmur A, Glatter O. Characterization and potential applications of nanostructured aqueous dispersions. Adv Colloid Interface Sci. 2009; 147:333-342.

16. Avachat AM, Parpani SS. Formulation and development of bicontinuous nanostructured liquid crystalline particles of efavirenz. Colloids Surf B Biointerfaces. 2015;126:87-97.

17. Budhian A, Siegel SJ, Winey KI. Controlling the in vitro release profiles for a system of haloperidol-loaded PLGA nanoparticles. Int J Pharm. 2008;346(1-2):151-159.

18. Hirsjärvi S, Sancey L, Dufort S, et al. Effect of particle size on the biodistribution of lipid nanocapsules: comparison between nuclear and fluorescence imaging and counting. Eur J Pharm. 2013;453(2): 594-600.

19. Tian T, Cai XJ, Zhu H. Puerarin, an isoflavone compound extracted from Gegen (Radix Puerariae Lobatae), modulates sclera remodeling caused by extremely low frequency electromagnetic fields. $J$ Tradit Chin Med. 2016;36(5):678-682.

20. Zhang H, Song Y, Li ZY, Zhang T, Zeng L. Evaluation of breviscapine on prevention of experimentally induced abdominal adhesions in rats. Am J Surg. 2016;211(6):1143-1152.

21. Wu XM, Chu JL, Xu TT, He BF. Isolation, identification and pharmacokinetic analysis of fructosyl puerarins from enzymatic glycosylation. J Chromatogr B Analyt Technol Biomed Life Sci. 2013;935:70-74.

22. Zhu YH, Jiang YQ, Liu ZH, Luo X, Wu Z. [The affect of Erigeron Breviscapus (Vant.) Hand-Mazz on axoplasmic transport of optic nerve in rats with experimentally elevated intraocular pressure]. Zhonghua Yan Ke Za Zhi. 2000;36(4):289-291. Chinese.

23. Shaikh J, Ankola DD, Beniwal V, Singh D, Kumar MN. Nanoparticle encapsulation improves oral bioavailability of curcumin by at least 9-fold when compared to curcumin administered with piperine as absorption enhancer. Eur J Pharm Sci. 2009;37(3-4):223-230.

24. Hao J, Wang X, Bi Y, et al. Fabrication of a composite system combining solid lipid nanoparticles and thermosensitive hydrogel for challenging ophthalmic drug delivery. Colloids Surf B Biointerfaces. 2014;114:111-120.

25. Chen HD, Pan H, Li PP, et al. The potential use of novel chitosan-coated deformable liposomes in an ocular drug delivery system. Colloids Surf B Biointerfaces. 2016;143(1):455-462. 
26. Gan L, Han S, Shen J, et al. Self-assembled liquid crystalline nanoparticles as a novel ophthalmic delivery system for dexamethasone: improving preocular retention and ocular bioavailability. Int J Pharm. 2010;396:179-187.

27. Wu HY, Liu ZD, Peng JJ, et al. Design and evaluation of baicalincontaining in situ $\mathrm{pH}$-triggered gelling system for sustained ophthalmic drug delivery. Int J Pharm. 2011;410(1-2):31-40.

28. Gratieri T, Gelfuso GM, de Freitas O, Rocha EM, Lopez RF. Enhancing and sustaining the topical ocular delivery of fluconazole using chitosan solution and poloxamer/chitosan in situ forming gel. Eur J Pharm Biopharm. 2011;79(2):320-327.

29. Adelli GR, Balguri SP, Punyamurthula N, Bhagav P, Majumdar S Development and evaluation of prolonged release topical indomethacin formulations for ocular inflammation. Invest Ophthalmol Vis Sci. 2014;55(13):463.

30. Tieleman DP, Berendsen HJ, Sansom MS. An alamethicin channel in a bilayer: molecular dynamics simulations. Biophys J. 1999;76: 1757-1769.

31. Schüttelkopf AW, Van Aalten DM. PRODRG: a tool for high-throughput crystallography of protein-ligand complexes. Acta Crystallogr D Biol Crystallogr. 2004;60:1355-1363.

32. Roux B. The calculation of the potential of mean force using computer simulations. Comput Phys Commun. 1995;91:275-282.

33. Marrink SJ, Berendse HJC. Simulation of water transport through a lipid membrane. J Phys Chem. 1994;98:4155-4168.

34. Kumar S, Bouzida D, Swendsen RH, Kollman PA, Rosenberg JM. The weighted histogram analysis method for free-energy calculations on biomolecules. I. The method. J Comput Chem. 1992;13:1011-1021.

35. Hub JS, Groot BL, van der Spoel D. g-wham. A free weighted histogram analysis implementation including robust error and autocorrelation estimates. J Chem Theory Comput. 2010;6:3713-3720.

36. Mehrabani J, Noaparast M, Mousavi S, Dehghan R, Ghorbani A. Process optimization and modelling of sphalerite flotation from a low-grade $\mathrm{Zn}-\mathrm{Pb}$ ore using response surface methodology. Sep Purif Technol. 2010;72:242-249.

37. Aslan N. Application of response surface methodology and central composite rotatable design for modeling the influence of some operating variables of a multi-gravity separator for coal cleaning. Fuel. 2007; 86:769-776.

38. Singh J, Chhabra G, Pathak K. Development of acetazolamide-loaded, $\mathrm{pH}$-triggered polymeric nanoparticulate in situ gel for sustained ocular delivery: in vitro ex vivo evaluation and pharmacodynamic study. Drug Dev Ind Pharm. 2013;40:1223-1232.
39. Luo Q, Lin T, Zhang CY, et al. A novel glyceryl monoolein-bearing cubosomes for gambogenic acid: preparation, cytotoxicity and intracellular uptake. Int J Pharm. 2015;493:30-39.

40. Lu C, Lu Y, Chen J, Zhang W, Wu W. Synchronized and sustained release of multiple components in silymarin from erodible glyceryl monostearate matrix system. Eur J Pharm Biopharm. 2007;66(2):201-209.

41. Xu HM, Li Z, Pan H, et al. A novel bi-layer ascending release osmotic pump tablet: in vitro investigation and in vivo investigation in pharmacokinetic study and IVIVC evaluation. Int J Pharm. 2013;458(1): 181-187.

42. Li QP, You J, Wang YS, Zhao Y, Yang L, Cui F. Simultaneous release of index components in Gegen Qinlian pellets. J Chin Tradit Herbal Drugs. 2006;01:40-44.

43. Fuente M, Ravina M, Paolicelli P, Sanchez A, Seijo B, Alonso MJ. Chitosan-based nanostructures: a delivery platform for ocular therapeutics. Adv Drug Deliv Rev. 2010;62:100-117.

44. Alany RG, Rades T, Nicoll J, Tucker IG, Davies NM. W/O microemulsions for ocular delivery: evaluation of ocular irritation and precorneal retention. J Control Release. 2006;111:145-152.

45. Liu R, Liu ZD, Zhang CG, Zhang BL. Gelucire44/14 as a novel absorption enhancer for drugs with different hydrophilicities: in vitro and in vivo improvement on transcorneal permeation. J Pharm Sci. 2011; 100:3186-3195.

46. Liu R, Sun L, Fang S, et al. Thermosensitive in situ nanogel as ophthalmic delivery system of curcumin: development, characterization, in vitro permeation and in vivo pharmacokinetic studies. Pharm Dev Technol. 2015;29:1-7.

47. Aoki S, Mizote H, Minamoto A, Suzuki M, Mishima HK, Tanaka H. Systemic FK506 improved tear secretion in dry eye associated with chronic graft versus host disease. Br J Ophthalmol. 2005;89:243-244.

48. Wang J, Ji M, Hua WY, Dai DZ. Development of puerarin. Adv Pharm Sci. 2003;27(2):70-74.

49. Kirschner DE, Hunt CA, Marino S, Fallahi-Sichani M, Linderman JJ. Tuneable resolution as a systems biology approach for multi-scale, multi-compartment computational models. Wiley Interdiscip Rev Syst Biol Med. 2014;6(4):289-309.

50. Berg EL. Systems biology in drug discovery and development. Nat Biotechnol. 2004;22:1253-1259.

51. Materi W, Wishart DS. Computational systems biology in drug discovery and development: methods and applications. Drug Discov Today. 2007; 12:295-303.
International Journal of Nanomedicine

\section{Publish your work in this journal}

The International Journal of Nanomedicine is an international, peerreviewed journal focusing on the application of nanotechnology in diagnostics, therapeutics, and drug delivery systems throughout the biomedical field. This journal is indexed on PubMed Central, MedLine, CAS, SciSearch $®$, Current Contents $\AA /$ Clinical Medicine,

\section{Dovepress}

Journal Citation Reports/Science Edition, EMBase, Scopus and the Elsevier Bibliographic databases. The manuscript management system is completely online and includes a very quick and fair peer-review system, which is all easy to use. Visit http://www.dovepress.com/ testimonials.php to read real quotes from published authors. 